\title{
Refractory hypotension induced by Sheehan syndrome with pituitary crisis: A case report
}

\author{
LU LIANG $^{1 *}$, JIN-BO LIU $^{1 *}$, FU-QIN CHEN ${ }^{1}$, JING ZHAO $^{2}$ and XIAO-LI ZHANG ${ }^{1}$ \\ Departments of ${ }^{1}$ Endocrinology and ${ }^{2}$ Cardiology, Qilu Hospital of Shandong University, \\ Jinan, Shandong 250000, P.R. China
}

Received December 5, 2015; Accepted December 19, 2016

DOI: $10.3892 /$ etm.2017.4188

\begin{abstract}
The present study reported on an unusual case of refractory hypotension induced by Sheehan syndrome with pituitary crisis and explored the causes and treatment of hypotension in hypopiptuitarism. Refractory hypotension is a rare and severe complication of longstanding hypopituitarism induced by Sheehan syndrome with pituitary crisis. A 48-year-old Chinese woman with pituitary crisis due to Sheehan syndrome developed refractory hypotension and received longstanding vasopressor blood pressure support and hormone replacement therapy. Besides normalized blood pressure, echocardiography over 3 months revealed partial reversibility of her cardiac function with hormone replacement therapy. Consistent numerous studies reviewed, hormone replacement therapy may improve cardiac function in patients with cardiomyopathy linked to Sheehan syndrome. A retrospective study of 77 cases with hypopituitarism encountered at Qilu Hospital of Shandong University (Jinan, China) was also performed and the incidence rate of hypotension was analyzed, revealing that the present case was the first of refractory hypotension induced by Sheehan syndrome with pituitary crisis at this institution in 16 years.
\end{abstract}

\section{Introduction}

Sheehan syndrome is characterized by hypopituitarism, which is due to ischemic necrosis of the pituitary gland secondary to postpartum hemorrhage (1). Major manifestations include failure to lactate, breast atrophy, secondary amenorrhea, genital and axillary hair loss, dry skin, hypopigmentation and other evidence of hypopituitarism. It can also present

Correspondence to: Professor Xiao-Li Zhang, Department of Endocrinology, Qilu Hospital of Shandong University, 107 Wenhua West Road, Lixia, Jinan, Shandong 250000, P.R. China

E-mail: sallyzh66@sina.com

*Contributed equally

Key words: refractory hypotension, Sheehan syndrome, pituitary crisis, hypopituitarism, hormone replacement therapy acutely with circulatory collapse, congestive cardiac failure and hypotension. Hypotension can be quickly corrected under hormone and volume replacement therapy. The present study reported on a case of Sheehan syndrome in a 48-year-old Chinese woman who had refractory hypotension and required longstanding vasopressor blood pressure support and hormone replacement therapy, which is rarely reported in the literature. The causes of refractory hypotension have been attributed to decreased cardiac output owing to cardiomyopathy and hypovolemia arising from hypoproteinemia. After three months, her blood pressure remained at levels of $\sim 110 / 70 \mathrm{mmHg}$ and her cardiac function partly reversed with hydrocortisone and levothyroxine replacement therapy.

\section{Case report}

A 48-year-old Chinese woman was admitted to the emergency department of Qilu Hospital of Shandong University (Jinan, China) with progressive chest distress and dyspnea for two weeks after a common cold. She had been suffering from long-term chronic symptoms, including fatigue, anorexia and light-headedness with a history of Sheehan syndrome without therapy. The patient had no history of cardiac disease or diabetes mellitus. In addition, the patient stopped menstruating after her second pregnancy 20 years previously.

Physical examination revealed the following: Mild hypothermia (axillary temperature, $35.8^{\circ} \mathrm{C}$ ), low blood pressure $(74 / 49 \mathrm{mmHg})$ and a pulse rate of 40 beats/min. Pale skin, facial edema and cool extremities with significant pitting edema of lower limbs were observed. Breast atrophy and sparse axillary and pubic hair were striking features. Lung examination revealed a moist rale in the bilateral lung bases with a respiratory rate of 20 breaths/min, muffled heart sounds and bradycardia were found in the cardiac auscultation area. Neurologically, the patient was without any focal signs.

Postpartum hypopituitarism had been identified and endocrine examination was performed (Table I). In addition to insulin-like growth factor-1 (IGF-1) deficiency, the patient's pituitary-thyroid, pituitary-gonadal and pituitary adrenal axes were dysfunctional and magnetic resonance imaging of the pituitary gland revealed an empty sella, indicating anterior hypopituitarism resulting from postpartum hemorrhage. Biochemical parameters at baseline and during follow-up are presented in Table II. A chest computed tomography (CT) 
Table I. Endocrine levels at baseline and follow-up.

\begin{tabular}{|c|c|c|c|c|}
\hline \multirow[b]{2}{*}{ Parameter } & \multicolumn{2}{|c|}{ During hospital } & \multirow{2}{*}{$\frac{\begin{array}{c}\text { After } \\
\text { discharge }\end{array}}{3 \text { months }}$} & \multirow[b]{2}{*}{ Normal range } \\
\hline & Baseline & Day 21 & & \\
\hline Free triiodothyronine $(\mathrm{pmol} / \mathrm{l})$ & $<1.54$ & 2.40 & 4.12 & $2.63-5.70$ \\
\hline Free thyroxine $(\mathrm{pmol} / \mathrm{l})$ & $<5.15$ & 10.41 & 27.06 & $9.01-19.05$ \\
\hline Thyroid-stimulating hormone $(\mu \mathrm{IU} / \mathrm{ml})$ & 0.36 & 0.26 & 0.04 & $0.35-4.94$ \\
\hline Cortisol $(\mu \mathrm{g} / \mathrm{dl})$ & 1.3 & & & $8.7-22.4$ \\
\hline Adrenocorticotrophic hormone (pg/ml) & 1.6 & & & $4.7-48.8$ \\
\hline Luteinizing hormone (mIU/ml) & 0.55 & & & $7.7-58.5$ \\
\hline Follicle-stimulating hormone (mIU/ml) & 0.34 & & & $25.8-134.8$ \\
\hline Estradiol $(\mathrm{pg} / \mathrm{ml})$ & 12.17 & & & $10-39.5$ \\
\hline Prolactin $(\mathrm{ng} / \mathrm{ml})$ & 0.31 & & & $3.4-24.1$ \\
\hline Insulin-like growth factor-1 (ng/ml) & 5.2 & & & $60-350$ \\
\hline Growth hormone $(\mathrm{ng} / \mathrm{ml})$ & 0.027 & & & $0.01-5.22$ \\
\hline
\end{tabular}

Table II. Biochemical parameters at baseline and follow-up.

\begin{tabular}{|c|c|c|c|c|c|c|c|c|}
\hline \multirow[b]{2}{*}{ Factors } & \multicolumn{6}{|c|}{ During hospital } & \multirow{2}{*}{$\frac{\begin{array}{c}\text { After } \\
\text { discharge }\end{array}}{1 \text { month }}$} & \multirow[b]{2}{*}{ Normal range } \\
\hline & Day 1 & Day 5 & Day 8 & Day 15 & Day 17 & Day 25 & & \\
\hline White blood cells $\left(\mathrm{x} 10^{9} / 1\right)$ & 7.75 & 15.61 & 13.99 & 10.87 & 9.40 & 6.60 & 6.92 & $3.5-9.5$ \\
\hline Neutrophils (\%) & 74.1 & 93.7 & 93.9 & 91.1 & 89.8 & 78.2 & 75.6 & $40-75$ \\
\hline Red blood cells $\left(\times 10^{12} / 1\right)$ & 3.43 & 3.24 & 4.31 & 3.74 & 3.62 & 3.55 & 3.42 & $3.8-5.1$ \\
\hline Hemoglobin $(\mathrm{g} / \mathrm{dl})$ & 103 & 101 & 137 & 117 & 112 & 110 & 108 & $115-150$ \\
\hline Platelets $\left(\times 10^{9} / 1\right)$ & 209 & 118 & 121 & 175 & 193 & 172 & 224 & $125-350$ \\
\hline Alanine transaminase (U/l) & 39 & 308 & 356 & 53 & 42 & 18 & 13 & $7-40$ \\
\hline Aspartate transaminase $(\mathrm{U} / \mathrm{l})$ & 68 & 399 & 297 & 44 & 41 & 19 & 18 & $13-35$ \\
\hline Total protein $(\mathrm{g} / \mathrm{l})$ & 72.0 & 46.0 & - & 47.6 & 48.1 & 50.6 & 65.7 & $60-85$ \\
\hline Albumin $(\mathrm{g} / \mathrm{l})$ & 45.0 & 30.4 & 28.8 & 32.6 & 30.7 & 32.9 & 43.2 & $40-55$ \\
\hline Blood urea nitrogen (mmol/l) & 13.3 & 8.9 & 10.0 & 11.4 & 10.9 & 14.1 & 11.1 & $2.3-7.8$ \\
\hline Creatinine $(\mu \mathrm{mol} / \mathrm{l})$ & 119 & 125 & 129 & 121 & 114 & 99 & 99 & $53-97$ \\
\hline Natrium (mmol/l) & 136 & 144 & 143 & 144 & 143 & 139 & 141 & $137-147$ \\
\hline Potassium (mmol/l) & 2.60 & 3.85 & 3.78 & 3.78 & 2.57 & 4.96 & 3.07 & $3.5-5.3$ \\
\hline Fasting blood glucose (mmol/l) & 4.70 & 9.15 & - & 8.27 & - & 6.80 & 4.43 & 3.9-6.1 \\
\hline Cholesterol $(\mathrm{mmol} / \mathrm{l})$ & 4.18 & - & - & - & - & - & 5.03 & $2.80-6.00$ \\
\hline Triglyceride (mmol/l) & 1.5 & - & - & - & - & - & 0.64 & $0.30-1.70$ \\
\hline LDL-C $(\mathrm{mmol} / \mathrm{l})$ & 2.45 & - & - & - & - & - & 2.95 & $1.00-3.37$ \\
\hline HDL-C (mmol/l) & 0.83 & - & - & - & - & - & 1.78 & $0.80-2.00$ \\
\hline
\end{tabular}

LDL-C, Low density lipoprotein cholesterin; HDL-C, high density lipoprotein cholesterin.

scan showed ill-defined ground glass opacity in the left lower lobe due to pulmonary consolidation. The abdominal CT scan was normal. An echocardiogram showed left ventricular (LV) global hypokinesis with decreased LV ejection fraction (LVEF), a medium amount of pericardial effusion and enlarged left atrium and right ventricle (Table III). Mask oxygen inhalation and anti-infective treatment (intravenous drip of meropenem and levofloxacin for two weeks) were initiated to improve respiratory symptoms. Along with exacerbation of congestive heart failure and renal and hepatic injuries, the patient was treated with volume replacement, digoxin $(0.125 \mathrm{mg} / \mathrm{dat})$ and dopamine $(3-5 \mu \mathrm{g} / \mathrm{min} / \mathrm{kg})$ to raise the blood pressure, albumin infusion to increase colloid osmotic pressure, furosemide injection to relieve edema as well as further symptomatic treatments. Initially, the patient was given a stress dose of intravenous hydrocortisone 
Table III. Indexes for cardiac function at baseline and follow-up.

\begin{tabular}{|c|c|c|c|c|c|c|c|}
\hline \multirow[b]{2}{*}{ Factor } & \multicolumn{4}{|c|}{ During hospital } & \multicolumn{2}{|c|}{ After discharge } & \multirow[b]{2}{*}{ Normal range } \\
\hline & Day 1 & Day 5 & Day 17 & Day 25 & 1 month & 3 months & \\
\hline \multicolumn{8}{|l|}{ Cardiac enzymes } \\
\hline Troponin-I (ng/ml) & 0.01 & 0.23 & 0.11 & 0.05 & 0.02 & - & $0-0.06$ \\
\hline CK-MB (ng/ml) & 8.0 & 4.7 & 4.6 & 2.1 & 2.2 & - & $0.3-4.0$ \\
\hline Nt-proBNP (pg/ml) & 548 & - & - & - & - & - & $<125$ \\
\hline \multicolumn{8}{|l|}{ Electrocardiogram } \\
\hline Heart rate (beats/min) & 40 & 111 & 63 & 65 & 65 & 77 & $60-100$ \\
\hline PR interval & 200 & 130 & 196 & 160 & 196 & 190 & $120-200$ \\
\hline QRS duration (msec) & 78 & 61 & 83 & 68 & 80 & 78 & $<120$ \\
\hline T wave & Flat & Flat & Flat & Flat & Flat & Normal & Normal \\
\hline QT/QTc (msec) & $600 / 490$ & $292 / 358$ & $362 / 402$ & $180 / 225$ & $494 / 405$ & $352 / 384$ & Variable/350-440 \\
\hline Low voltage & $\begin{array}{l}\text { Limb } \\
\text { leads }\end{array}$ & $\begin{array}{l}\text { Limb } \\
\text { leads }\end{array}$ & $\begin{array}{l}\text { Limb } \\
\text { leads }\end{array}$ & $\begin{array}{l}\text { Limb } \\
\text { leads }\end{array}$ & $\begin{array}{l}\text { Limb } \\
\text { leads }\end{array}$ & None & None \\
\hline \multicolumn{8}{|l|}{ Echocardiography } \\
\hline LA dimension (mm) & 40 & - & - & 35 & - & 34 & $<35$ \\
\hline $\mathrm{RV}$ dimension (mm) & 30 & - & - & 26 & - & 23 & $<25$ \\
\hline $\operatorname{LVEF}(\%)$ & 30 & - & - & 55 & - & 64 & $>50$ \\
\hline LV motion & $\begin{array}{l}\text { Abnormal } \\
\text { diastolic } \\
\text { hypokinesis }\end{array}$ & - & - & $\begin{array}{l}\text { Abnormal } \\
\text { diastolic } \\
\text { filling }\end{array}$ & - & $\begin{array}{l}\text { Abnormal } \\
\text { diastolic } \\
\text { filling }\end{array}$ & Normal \\
\hline Tricuspid regurgitation & Severe-moderate & - & - & Moderate & - & Mild & Normal \\
\hline Pericardial effusion & $\begin{array}{l}\text { Medium } \\
\text { amount }\end{array}$ & - & - & $\begin{array}{l}\text { Medium } \\
\text { amount }\end{array}$ & - & $\begin{array}{l}\text { Small } \\
\text { amount }\end{array}$ & None \\
\hline
\end{tabular}

CK, creatine kinase; BNP, B-type natriuretic peptide; LVEF, left ventricle ejection fraction; LA, left atrium.

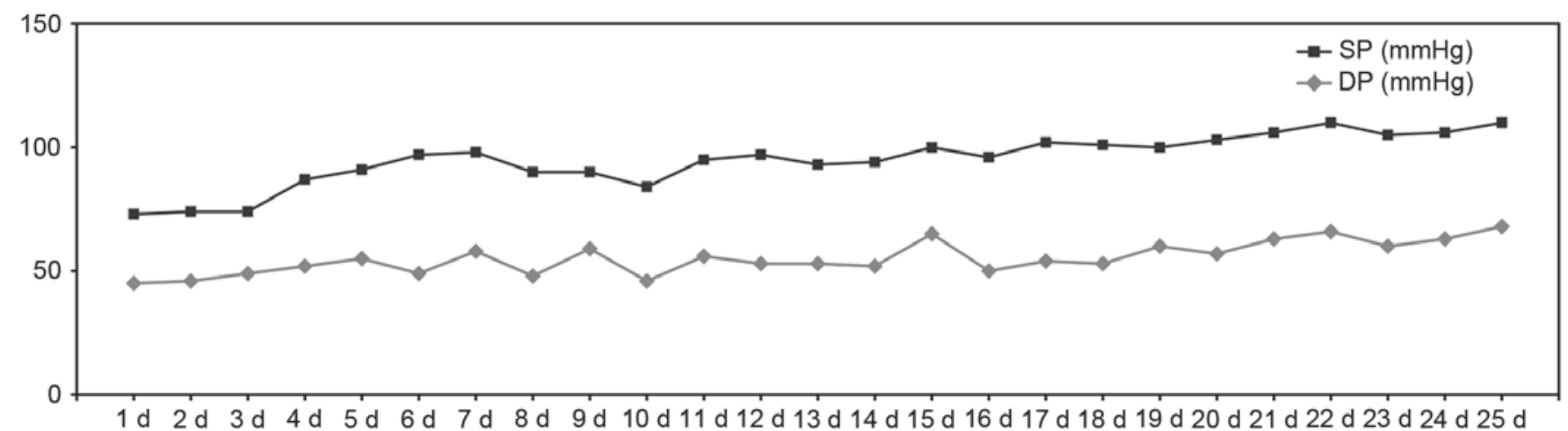

Figure 1. Blood pressure levels ( $\mathrm{mmHg}$ ) in the patient over the course of the treatment. SP, systolic blood pressure; DP, diastolic blood pressure; $\mathrm{d}$, day(s).

(50 $\mathrm{mg} / 6 \mathrm{~h}$ ) with a gradual reduction to $100 \mathrm{mg} / \mathrm{day}$, followed by a low dose of oral levothyroxine ( $25 \mu \mathrm{g}$ /day), which was increased to $100 \mu \mathrm{g} /$ day. Due to the patient's low and erratic blood pressure, she was fully weaned off vasopressor blood pressure support over 20 days, with blood pressure remaining at levels of around 90/60 $\mathrm{mmHg}$ (Fig. 1). Moreover, the patient's general health condition markedly improved, dyspnea eased, chest distress disappeared, edema decreased and fatigue alleviated. Abnormal biochemical indexes were almost restored to normal levels. A later echocardiography showed dramatic changes in LVEF from 30 to 55\%, with a decrease of pericardial effusion and mild tricuspid regurgitation (Table III). The patient was discharged on hydrocortisone (40 mg/day) and levothyroxine (100 $\mu \mathrm{g} /$ day) 28 days after being admitted.

At follow-up 3 months after discharge, a repeat echocardiogram showed LV filling disturbance with a continuous increase in LVEF (64\%) as well as normal cardiac size, and blood pressure was increased to $110 / 70 \mathrm{mmHg}$ with glucocorticoid and levothyroxine replacement therapy. The dose of 
levothyroxine was decreased from 100 to $75 \mu \mathrm{g}$ as a result of a higher free thyroxine level.

\section{Discussion}

Acute pituitary insufficiency, also called pituitary crisis, is a life-threatening condition following a period of non-specific symptoms due to chronic pituitary insufficiency, the causes of which involve electrolyte imbalance, infection, trauma or other forms of stress. Volume depletion and low cardiac output are common in acute pituitary deficiency and recovery of normal cardiovascular status is rapidly achieved under hormone and volume replacement therapy. Vesely et al (2) reported a case of post-herpes encephalitic anterior pituitary insufficiency (dysfunction of pituitary-thyroid and pituitary-gonadal axis) with hypothermia and hypotension in a 49-year-old man, and his blood pressure of $90 / 60 \mathrm{mmHg}$ quickly returned to normal after thyroid hormone replacement therapy. However, the patient of the present study experienced refractory hypotension and required longstanding vasopressor blood pressure support and hormone replacement therapy, which was rarely reported in the literature. Retrospective analysis of 77 retrieved cases of Sheehan syndrome at Qilu Hospital (Jinan, China) from 1999 to 2015 revealed that patients with hypotension accounted for $29.9 \%$ (23 cases), whose normalization of blood pressure almost generally occurred on the third or fourth day of treatment, after hormone replacement and correction of hyponatremia.

In the present case, one cause of severe hypotension was closely associated with hypoproteinemia owing to chronic malnutrition and liver damage. Albumin can expand fluid and maintain a stable plasma colloid osmotic pressure, while hypoproteinemia impairs water balance, increasing the likelihood of hypovolemia. Moreover, albumin infusion combined with diuretic injection helped to ease myocardial edema and pericardial effusion, which are beneficial for cardiac function.

Initially, septic shock resulting in hypotension could not be excluded, as indicated by an increased white blood cell count and neutrophils, respiratory symptoms and chest radiological findings. After potent fluoroquinolones (levofloxacin) and cabapenems (meropenem) had been administered for two weeks, respiratory symptoms were obviously remitted. However, the white blood cell count did not decrease, which was associated with the intravenous infusion of hydrocortisone (50 mg/6 h). With the extenuation of hydrocortisone, the white blood cell count returned to normal. Infectious factors were no longer considered, as the blood pressure was not markedly elevated.

During the evaluation, symptomatic heart failure and elevated cardiac enzymes raised the suspicion of myocardial ischemia or an acute infraction resulting in hypotension. It has been reported that endocrine disorders lead to abnormal lipid and glucose metabolism and are therefore implicated in the genesis of acute coronary syndrome (ACS). However, this possibility was quickly ruled out based on the following points: i) Cardiac markers are not specific for ACS and only resemble a manifestation of myocardial damage to a certain extent; ii) serum lipid levels, a risky factor for assessing cardiovascular disease, were in the normal range; iii) nonspecific and abnormal electrocardiogram (ECG) patterns, such as sinus bradycardia, prolonged QT intervals, low limb lead voltage and abnormal $\mathrm{T}$ wave, suggested a correlation with metabolic disease; iv) compared with the original ECG, there were no dynamic changes indicating ACS; v) the patient had no history of cardiac disease or diabetes mellitus; vi) an echocardiogram showed LV global hypokinesis with decreased LVEF, which was consistent with cardiomyopathy (Table III). Therefore, the patient's refractory hypotension was attributed to cardiomyopathy.

Of all endocrine hormone deficiencies linked to cardiomyopathy, glucocorticoid, thyroid hormone and growth hormone deficiencies have major roles. The mechanisms responsible for the development of cardiomyopathy are varied: i) Thyroid hormone deficiency has a significant impact on myocardial injury, resulting in weakening of myocardial contraction and relaxation, decrease in cardiac output, and rhythm disturbances, through genomic and non-genomic effects (3-5). ii) Catecholamine overproduction during stress may be toxic to the myocardium, which is unprotected by inadequate glucocorticoids, impairing cardiac function; glucocorticoid deficiency disturbs the transport function of the membrane calcium pump, affecting myocardial contractility $(6,7)$. iii) Numerous experimental studies have demonstrated that growth hormone/ IGF-1 deficiency has a deleterious influence on cardiac growth, myocardial contractility and vascular system $(8,9)$. However, certain case studies have indicated that growth hormone deficiency has a minimal role in pathogenic mechanisms of cardiomyopathy $(10,11)$. Laway et al (10) described a case of cardiomyopathy linked to Sheehan syndrome and pulmonary tuberculosis in a 25-year-old women, whose cardiac function was completely restored after replacement therapy with glucocorticoid and levothyroxine.

On the basis of the abovementioned pathogenesis, the patient was initiated with a stress dose of hydrocortisone, followed by a small dose of oral levothyroxine under the state of high stress. Glucocorticoid is replaced prior to levothyroxine to avoid aggravating pituitary crisis. In contrast to previous patients, the patient of the present study was also given positive inotropic agents, digoxin and dopamine, which improved hemodynamics and increased cardiac pump function. Her blood pressure did not return to normal until vasopressor blood pressure support and hormone replacement therapy were provided for 20 days. It is speculated that a longstanding adverse influence on cardiac function may be the primary cause of refractory hypotension. The patient was discharged whilst receiving a physiological dosage of hydrocortisone and levothyroxine. At 3-month follow-up, cardiac function was partly reversed with preserved LVEF, normal cardiac size, small pericardial effusion and abnormal LV diastolic filling.

The definite reason why the patient's hormone-induced cardiac dysfunction was not completely resolved remains elusive. One explanation that long-standing hormone deficiencies drive the irreversible change of myocardial damage appears to not be dependable $(11,12)$. Bao and Fisher (11) reported on a 35-year-old woman with long-standing hypopituitarism for 15 years and heart failure for at least 10 years with severely compromised cardiac function. At 9 months after discharge, echocardiography showed completely normalized cardiac function without growth hormone replacement (11). Similarly, Kissell et al (12) reported on a 40-year-old patient 
with cardiogenic shock due to non-ischemic cardiomyopathy induced by severe anterior hypopituitarism initially treated with levothyroxine and hydocortisone replacement therapy, who had 20 years of history of undiagnosed Sheehan syndrome. Eighteen months later, echocardiography revealed that LVEF was partially reversed (12). One hypothesis is that GH deficiency is involved in pathogenic mechanisms of cardiomyopathy, although there is a possibility of complete recovery of cardiac function without growth hormone replacement $(10,11)$. In the present case, the irreversibility of cardiac damage may have been associated with the short duration of hormone replacement therapy, while there is a possibility that cardiomyopathy may be completely reversed in the future.

The importance of a rapid diagnosis of Sheehan syndrome, particularly intercurrent hypopituitary crisis, should be emphasized, as otherwise, treatment is delayed and patients remain in a critical condition. Kaufmann et al (13) reported on a 52-year-old woman who developed hypopituitary crisis with severe hypotension and coma secondary to unrecognized chronic anterior hypophysitis. Unfortunately, she succumbed to refractory cardiac arrest without prompt hormone replacement treatment. In the present case, the important information that the patient had a history of Sheehan syndrome was available, which provided a hint for selecting an appropriate treatment.

In conclusion, a prompt diagnosis and specific treatment of Sheehan syndrome is vital. The present case illustrated that refractory hypotension is a rare and serious complication of longstanding hypopituitarism induced by Sheehan syndrome with pituitary crisis, of which volume depletion and low cardiac output are common pathological mechanisms. The case also exemplified that glucocorticoid, thyroid hormone and growth hormone deficiencies may contribute to cardiomyopathy by varying degrees, and cardiac function may be restored with hormone replacement therapy.

\section{References}

1. Shivaprasad C: Sheehan syndrome's syndrome: Newer advances. Indian J Endocrinol Metab 15 (Suppl 3): S203-S207, 2011.

2. Vesely DL, Mastrandrea P, Samson C, Argyelan G and Charvit S: Post-herpes encephalitic anterior pituitary insufficiency with hypothermia and hypotension. Am J Med Sci 320: 273-277, 2000.

3. Vargas-Uricoechea H and Sierra-Torres $\mathrm{CH}$ : Thyroid hormones and the heart. Horm Mol Biol Clin Invest 18: 15-26, 2014

4. Galli E, Pingitore A and lervasi G: The role ofthyroid hormone in the pathophysiology of heart failure: Clinical evidence. Heart Fail Rev 15: 155-169, 2010.

5. Schmidt-Ott UM and Ascheim DD: Thyroid hormone and heart failure. Curr Heart Fail Rep 3: 114-119, 2006.

6. Cleghorn RA: Cardiovascular failure in experimental adrenal insufficiency: A history revival. Perspect Biol Med 27: 135-155, 1983.

7. Narayanan $\mathrm{N}$ : Effects of adrenalectomy and in vivo administration of dexamethasone on ATP-dependent calcium accumulation by sarcoplasmic reticulum from rat heart. J Mol Cell Cardiol 15: $7-15,1983$.

8. Castellano G, Affuso F, Conza PD and Fazio S: The GH/IGF-1 axis and heart failure. Curr Cardiol Rev 5: 203-215, 2009.

9. Lombardi G, Di Somma C, Grasso LF, Savanelli MC, Colao A and Pivonello R: The cardiovascular system in growth hormone excess and growth hormone deficiency. J Endocrinol Invest 35: 1021-1029, 2012.

10. Laway BA, Alai MS, Gojwari T, Ganie MA and Zargar AH: Sheehan syndrome with reversible dilated cardiomyopathy. Ann Saudi Med 30: 321-324, 2010.

11. Bao SS and Fisher SJ: Repairing a 'broken heart' with hormone replacement therapy: Case report of cardiogenic shock due to undiagnosed pituitary insufficiency. Endocr Pract 18: e26-e31, 2012.

12. Kissell N, Mudd JO, Gelow JM, Chong LE and Yuen KC: Cardiogenic shock due to non-ischemic cardiomyopathy induced by severe anterior hypopituitarism. Endocr Pract: Nov 4, 2014 (Epub ahead of print).

13. Kaufmann P, Lax SF, Radner H, Eber B, Leuger A and Smolle KH: Severe hypotension and coma secondary to unrecognized chronic anterior hypophysitis. Intensive Care Med 21: 847-849, 1995. 